\title{
Handling Location Uncertainty in Probabilistic Location-Dependent Queries
}

\author{
Carlos Bobed $^{\mathrm{a}, 1, *}$, Jorge Bernad ${ }^{\mathrm{a}, 1}$, Sergio Ilarri $^{\mathrm{a}}$, Eduardo Mena $^{\mathrm{a}}$ \\ ${ }^{a}$ Dept. of Computer Science $\mathcal{E}$ Systems Engineering \\ University of Zaragoza \\ 50018 Zaragoza, Spain
}

\begin{abstract}
Location-based services have motivated intensive research in the field of mobile computing, and particularly on location-dependent queries. Existing approaches usually assume that the location data are expressed at a fine geographic precision (physical coordinates such as GPS). However, many positioning mechanisms are subject to an inherent imprecision (e.g., the cell-id mechanism used in cellular networks can only determine the cell where a certain moving object is located). Moreover, even a GPS location can be subject to an error or be obfuscated for privacy reasons. Thus, moving objects can be considered to be associated to an uncertainty area where they can be located.

In this paper, we analyze the problem introduced by the imprecision of the location data available in the data sources by modelling them using uncertainty areas. To do so, we propose to use a higher-level representation of locations which includes uncertainty, formalizing the concept of uncertainty location granule. This allows us to consider probabilistic location-dependent queries, among which we will focus on probabilistic inside (range) constraints. The adopted model allows us to develop a systematic and efficient approach for processing this kind of queries. An experimental evaluation shows that these probabilistic queries can be supported efficiently.
\end{abstract}

Keywords:

Probabilistic Range Queries, Location-Dependent Queries, Uncertainty Management.

\section{Introduction}

Location-Based Services [34, 39] (LBSs) have motivated intensive research in the field of mobile computing. These services provide value-added data by considering the locations of the mobile users and other moving objects to offer more customized information. As a basic building block of LBSs, the efficient processing of location-dependent queries [23] (queries whose answer depends on the location of certain moving objects) is a key issue. As a sample location-dependent query, consider a user with a smartphone that wants to locate the available taxi cabs that are near her while she is walking home on a rainy day.

Most proposals on location-dependent query processing implicitly assume GPS locations for the objects in a scenario (e.g., $[8,16,17,23,30,33,35])$. However, in some cases we do not require location data at GPS resolution, and a coarser representation may be more appropriate. For example, a train tracking application may need to know in which city a train is currently located (its precise GPS coordinates may just be meaningless), and thus each city as a whole could be considered a different location. To model these situations, in $[5,21]$ we defined the

\footnotetext{
${ }^{*}$ Corresponding author

Email addresses: cbobed@unizar.es (Carlos Bobed), jbernad@unizar.es (Jorge Bernad), silarri@unizar .es (Sergio Ilarri), emena@unizar.es (Eduardo Mena)

${ }^{1}$ The first two authors contributed equally to this work.
}

concept of location granule as a set of physical locations with an associated semantics. Our proposal allows users to express queries and retrieve results based on the concept of "location" that they need to manage, which may correspond with GPS locations but, in general, it will be locations at a lower resolution (e.g., freeways, buildings, offices in a building, segments in a motorway, etc.). Thus, location granules represent areas with some semantics interesting for the user rather than just being plain granularity indications of location resolution or precision. Besides, managing location granules instead of precise geographic locations could also be interesting for privacy reasons, as a LBS should not track location information more precise than what is required to provide the service [3]; moreover, the chosen granularity may depend not only on the service required but also on the user preferences [36].

In our previous work [21], we proposed a query processing architecture based on a distributed infrastructure of different data sources, each one covering a specific area and managing the location information of all the moving objects within (as also proposed in works such as [22, 32]). However, the main problem of that approach was that we assumed that the data sources that store information about moving objects are able to provide the precise GPS locations of the moving objects, which is a strong assumption that we remove with this work. Thus, it is well-known that many positioning mechanisms have an inherent imprecision [43]; for example, the cell-id mechanism used in cellular networks can only determine the cell where a certain 
moving object is located [40], but not its precise location within the cell. Similarly, location imprecision is also unavoidable in object-tracking wireless sensor networks [29]. Moreover, even GPS coordinates can have an error [42]. Finally, for privacy reasons, artificial errors may be introduced in the locations of certain moving objects [27].

In this paper, we analyze the problem introduced by the imprecision of the location data available in the data sources, and consider probabilistic location-dependent queries using location granules to model location uncertainty. Thus, we extend our previous location model (based on location granules) by proposing the concept of uncertainty location granule (or just uncertainty granule) to model situations where the uncertainty is part of the nature of the problem. Intuitively, an uncertainty granule is a place or an area where an object can be located, together with a probability density function ( $p d f$ from now on) to know the likelihood that the object was in any point of this area. This extension allows us to consider probabilistic granule-based location-dependent queries, among which we focus in this paper on queries with inside constraints, i.e., constraints that are satisfied by objects located within a certain range around a given moving object. As an example, imagine that we want to monitor policemen that are located less than $r$ meters from a place where a certain criminal is currently located with a probability of at least $70 \%$; alternatively, for example, we might want to monitor all the policemen that may be within that radius and obtain the probability that they are actually within the radius. Let us note that we deal with uncertainty places (uncertain locations): 1) the place where the criminal (what we call the reference object) is located, and 2) the places where policemen (what we call the target objects) are located. We will call to this type of queries probabilistic inside queries. This type of queries can be considered a specialization for the spatial domain in $\mathbf{R}^{2}$ of fuzzy probabilistic range queries [38] or uncertain range queries [45]; the concept of probabilistic similarity join defined in [25] is also similar as well. While there have been many works on the processing of different types of queries in the presence of uncertainty $[6,9,13,24,28,31,46]$, there is a lack of an in-depth formal study of this type of queries, along with an efficient processing approach, where uncertainty is also considered for the queried position (as it can be the position of moving object) and which does not assume the availability of indexes of moving objects. Moreover, we provide an extensive experimental evaluation including simulated scenarios and mobile devices. In a sense, we follow a similar processing schema as in [9], where the authors propose a three-step query processing approach, namely, filtering (i.e., prune out objects with zero probabilities of being in the answer set), verification (i.e., calculate sufficient conditions - probability bounds- for an object to be or not to be in the answer set), and refinement (i.e., calculate the exact probabilities); our work completely focuses on second third steps, not being bound to any particular approach for the first one.

The main contributions of this paper are:

- We formalize the concept of uncertainty granule and its relationship with traditional location granules (granules without uncertainty).

- We analyze a type of query, that we call probabilistic inside query, and provide a method to solve them efficiently when the underlying probability density function is the uniform distribution. Note that, in fact, the uniform distribution is the case where the least information we have about the position of the objects, and it has been used in many previous works $[11,12,24,28]$.

- We perform an extensive experimental evaluation where the efficiency of the described method for the uniform distribution can be appreciated.

The structure of the rest of this paper is as follows. In Section 2, we present an overview of location granules, extend their basic definition to incorporate uncertainty, and formally define the problem we tackle in this paper. Then, in Section 3, we focus on probabilistic inside queries, and explain our approach to tag objects with the probabilities that they are part of the answer to the query. In Section 4, we focus on the case of uniform $p d f s$, providing a method to quickly determine whether an object belongs to the answer set for a given query. In Section 5, we present an experimental evaluation that shows that the probabilistic location-dependent queries studied in this paper can be supported efficiently. In Section 6, we present some related work. Finally, in Section 7 , we draw our conclusions and present some ideas for future work.

\section{Location Granules and Uncertainty}

In this section, we first provide a brief explanation of the notion of location granule. Then, we introduce the data model used to represent location granules and its extension to deal with uncertainty and imprecise locations. Finally, we formally define the type of range queries we deal with in this paper, to state the problem we address in latter sections. For better readability, we summarize in Table 1 the notations that will be used throughout the paper.

A location granule $[5,21]$ refers to one or more geographic areas which identify a set of GPS locations under a common name. As an example, it can be said that a given car is at a certain $<\mathrm{x}, \mathrm{y}\rangle$ location, but alternatively it can be stated, for example, that it is in the location granule Los Angeles (a location granule of type city), in the location granule California (a location granule of type state), or in the location granule USA (a location granule of type country), depending on the location granularity required (GPS, city, state, or country, respectively). This concept of semantic location is similar to the notion of place $[18,20]$ or spatial granule [4] proposed in the literature.

\subsection{Data Types: Uncertainty Location Granules}

The basic data model that we consider for location-dependent query processing with location granules is composed of three datatypes (see Table 2 for a summary): 
Table 2: Basic probabilistic data model: datatypes and main operators for location granules and uncertainty granules.

\begin{tabular}{|c|c|c|}
\hline Datatype & Tuple format & Operators \\
\hline Object (OB) & $<$ id, name, loc, class, otherAttr $>$ & representObject \\
\hline Location Granule (LG) & $<$ id, name, $G_{A}>$ & inGr: LG x GPS $\rightarrow$ Boolean \\
& & inGr: LG x OB $\rightarrow$ Boolean \\
& & inGr: $\mathcal{P}($ LG) $x$ GPS $\rightarrow$ Boolean \\
& & dist: GPS x LG $\rightarrow$ Real \\
& & distBtwGrs: LG x LG $\rightarrow$ Real \\
\hline Uncertainty Granule (UG) & $<$ id, $U G_{t}$, density function $>$ & inGrProb: LG x UG $\rightarrow$ Real \\
& & inGrProb: $\mathcal{P}($ LG) x UG $\rightarrow$ Real \\
& & distProb: LG x UG $\rightarrow$ PDF \\
& & distProb: UG x UG $\rightarrow$ PDF \\
\hline
\end{tabular}

Table 1: Notations in the paper.

\begin{tabular}{|c|c|}
\hline$G_{i}$ & location granule \\
\hline$E A\left(G_{i}\right)$ & $\begin{array}{l}\text { extended area of a location } \\
\text { granule }\end{array}$ \\
\hline$O_{i}=<i d, U G_{i}, p d f_{i}>$ & $\begin{array}{l}\text { uncertainty granule with as- } \\
\text { sociated uncertainty area } U G_{i} \\
\text { and probability density func- } \\
\text { tion } p d f_{i}\end{array}$ \\
\hline$P(O \in G)$ & $\begin{array}{l}\text { probability that the uncer- } \\
\text { tainty granule } O \text { is located in- } \\
\text { side the location granule } G\end{array}$ \\
\hline inGrProb $(O, G)$ & same as $P(O \in G)$ \\
\hline distProb $(G, O)(R)$ & $\begin{array}{l}\text { probability that the distance } \\
\text { between } O \text { and } G \text { is equal to } \\
R\end{array}$ \\
\hline $\begin{array}{l}P(\text { distProb }(G, O) \leq \\
R) \\
P\left(\text { distProb }\left(O^{\prime}, O\right) \leq\right. \\
R)\end{array}$ & $\begin{array}{l}\text { probability that the distance } \\
\text { between } G\left(O^{\prime}\right) \text { and } O \text { is less } \\
\text { or equal to } R\end{array}$ \\
\hline $\operatorname{probL}_{O_{r}}^{O_{t}}(R)$ & $\begin{array}{l}\text { same as } P\left(\text { distProb }\left(O_{t}, O_{r}\right) \leq\right. \\
R)\end{array}$ \\
\hline$B(\mathbf{l}, r)$ & $\begin{array}{l}\text { disk centered in } \mathbf{l} \in \mathbf{R}^{2} \text { with } \\
\text { radius } r \in \mathbf{R}\end{array}$ \\
\hline$f_{1}^{r}$ & $\begin{array}{l}\text { uniform density function over } \\
B(\mathbf{l}, r)\end{array}$ \\
\hline$f_{\mathbf{l}_{1}, \mathbf{l}_{2}}^{R_{1}, R_{2}}$ & $\begin{array}{l}\text { convolution product of } f_{\mathbf{l}_{1}}^{R_{1}} \\
\text { and } \bar{f}_{\mathbf{l}_{2}}^{R_{2}}\end{array}$ \\
\hline$A_{R_{1}}^{R_{2}}(x)$ & $R_{1}^{2} \arccos \left(\frac{R_{1}^{2}-R_{2}^{2}+x^{2}}{2 R_{1} x}\right)$ \\
\hline$T_{R_{1}, R_{2}}(x)$ & $\sqrt{4 R_{1}^{2} R_{2}^{2}-\left(R_{1}^{2}+R_{2}^{2}-x^{2}\right)^{2}}$ \\
\hline$C_{1}^{R_{1}, R_{2}}$ & $\begin{array}{l}\text { annulus centered at } \mathbf{I} \text { and ra- } \\
\text { dius } R_{1} \text { and } R_{2}\end{array}$ \\
\hline$C_{1}^{R_{1}, R_{2}}(\alpha, \beta)$ & $\begin{array}{l}\text { Portion of the annulus } C_{1}^{R_{1}, R_{2}} \\
\text { between an angle } \alpha \text { and } \beta\end{array}$ \\
\hline
\end{tabular}

- Objects are characterized by an internal (system-managed) identifier, a name, a GPS location (loc.x and loc.y), a class, and probably other attributes specific to their class.

- A location granule (or simply a granule) $G$ is a tuple (id, name, $G_{A}$ ) where id is an internal (system-managed) identifier, name is the name of the granule, and $G_{A} \subseteq \mathbf{R}^{2}$ is the area of the location granule. It provides three main kinds of operators: the in $\mathrm{Gr}$ operators (short for inGranule) returns a boolean indicating whether a certain GPS location or location granule is within a specified granule (or within a set of granules), the dist operator (distanceGranule) computes the distance between a provided GPS location and a granule, and distBtwGrs (distanceBetweenGranules) computes the distance between two granules. Different types of distances can be considered: the limits-distance (the minimum distance to the boundaries of the areas composing the granule), the centroiddistance (the distance to the centroids of the granules), and the outsideLimitsBased-distance (a variation of the limits-distance in which all the inner points of the granule are considered to be at zero distance). Unless otherwise indicated, we will use in this paper the limits-distance.

Up to this point, no special provisions have been made to consider location uncertainty in the data model. So, to deal with imprecise locations and probabilistic queries, we extend the basic model by introducing the notion of uncertainty granule.

Definition 1. Uncertainty Granule $(U G)$. An element $O_{t}$ of type Uncertainty Granule (in the following, type $U G$ ) is a tuple $O_{t}=<$ $i d, U G_{t}, p d f_{t}>$ composed by:

- an internal (system-managed) identifier id.

- $U G_{t} \subseteq \mathbf{R}^{2}$, which is an area or a discrete set of points; we will say that $U G_{t}$ is the uncertainty area of $O_{t}$.

- a function $p d f_{t} \in P D F$, the set of all probability density functions, whose interpretation depends on whether $U G_{t}$ is an area or a set of points: if $U G_{t}$ is an area, $p d f_{t}$ is a probability density function from $\mathbf{R}^{2}$ to $\mathbf{R}$ that represents 
the probability of an object being in each point of this area, and therefore, $\int_{U G_{t}} p d f_{t}=1$; if $U G_{t}$ is a discrete set of points, $p d f_{t}$ is a probability mass function such that $\sum_{x \in U G_{t}} p d f_{t}(x)=1$.

Without loss of generality, an element $x \in U G_{t}$ is said to be an instance of the uncertainty granule $O_{t}$ and we will denote it by $x \in O_{t}$.

Different probability density functions could be considered to define an uncertainty granule, depending on the information available as background knowledge. As an example, a uniform distribution could be assumed, which means that the object can be with the same probability in any location within the corresponding uncertainty granule. Of course, a specific probability density function could consist of different sub-functions to estimate the probability for each of the component areas (e.g., considering a uniform distribution within some of the areas and a Gaussian distribution in others).

An uncertainty granule can represent an imprecise location. For example, due to GPS errors, a car can be considered as an uncertainty granule $O_{c}=<i d, U G_{c}, p d f_{c}>$, where $U G_{c}$ is a disk of a given radius (the error radius), and $p d f_{c}$ is the uniform distribution over the disk $U G_{c}$.

From the previous definitions, it follows that when the system has no uncertainty about the location of a certain object, then its precise location can be defined by a particular case of uncertainty granule $<i d, U G_{t}, p d f_{t}>$ (see Definition 1) where $U G_{t}$ is equal to the precise location $\left(x_{0}, y_{0}\right)$ and $p d f_{t}$ is the following probability mass function:

$$
p d f(x, y)= \begin{cases}1 & \text { if }(x, y)=\left(x_{0}, y_{0}\right) \\ 0 & \text { otherwise }\end{cases}
$$

Finally, we also have to extend the model by adding new operators that relate location granules with uncertainty granules. The in $G r$ operators turns into inGrProb, which takes as input a granule (or a set of granules) and an uncertainty granule, and returns the probability that the imprecise location represented by the uncertainty granule is inside the location granule, that is, if $G$ is a location granule with associated area $G_{A}$, and $O_{t}$ is an uncertainty granule with associated $p d f p d f_{t}$, then:

$$
\operatorname{in} \operatorname{Gr} \operatorname{Prob}\left(G, O_{t}\right)=\int_{G_{A}} p d f_{t}
$$

We also use the notation $P\left(O_{t} \in G\right)$ (probability that $O_{t}$ is in $G$ ) for in $\operatorname{Gr} \operatorname{Prob}\left(G, O_{t}\right)$.

The other two operators that we introduce are: the distance between location granules and uncertainty granules, and the distance between uncertainty granules. The former operator takes as input a location granule $G$ and an uncertainty granule $O_{t}$ and returns a probability density function:

$$
\operatorname{distProb}\left(G, O_{t}\right)=f: \mathbf{R} \rightarrow \mathbf{R}
$$

such that $\operatorname{dist} \operatorname{Prob}\left(G, O_{t}\right)(R)$ represents the probability that the distance between the imprecise location represented by the uncertainty granule $O_{t}$ and the location granule $G$ is equal to $R$, that is:

$$
\operatorname{distProb}\left(G, O_{t}\right)(R)=P\left(\left\{x \in O_{t} \mid \operatorname{dist}(x, G)=R\right\}\right)
$$

Similarly, the operator $\operatorname{distProb}\left(O_{1}, O_{2}\right)$, where $O_{1}$ and $O_{2}$ are uncertainty granules, is defined by;

$$
\operatorname{distProb}\left(O_{1}, O_{2}\right)=f: \mathbf{R} \rightarrow \mathbf{R}
$$

and $\operatorname{dist} \operatorname{Prob}\left(O_{1}, O_{2}\right)(R)$ is the probability that the imprecise locations modeled by the uncertainty granules $O_{1}$ and $O_{2}$ are at distance $R$. Note that the distances involving uncertainty granules do not return a real number, but a function representing a probability density function.

We denote by $P(\operatorname{distProb}(G, O) \leq R$ ) (probability that $G$ and $O$ are not further than $R$ ) the value:

$$
P(\operatorname{distProb}(G, O) \leq R)=\int_{0}^{R} \operatorname{distProb}(G, O)(x) d x
$$

Similarly, we denote by $P\left(\operatorname{dist} \operatorname{Prob}\left(O_{1}, O_{2}\right) \leq R\right)$ the probability that the distance between $O_{1}$ and $O_{2}$ is less or equal than $R$.

\subsection{Probabilistic Inside Queries: Problem Statement}

Now that we have defined the data model we are using to represent objects and location granules with uncertain locations, we are able to define the probabilistic location-based constraints that we address in this paper.

Definition 2. Let $\mathcal{D}=\left\{O_{1}, \ldots, O_{n}\right\}$ be a set of uncertainty granules, $O_{r}$ be an uncertainty granule, and $R$ and $p$ be real numbers. A probabilistic inside query, $q\left(\mathcal{D}, O_{r}, R, p\right)$, returns the uncertainty granules of the set $\mathcal{D}$ that are no further than $R$ from the reference object $O_{r}$ with a probability greater or equal to $p$, that is:

$$
\begin{aligned}
& q\left(\mathcal{D}, O_{r}, R, p\right)= \\
& \quad\left\{O_{i} \in \mathcal{D} \mid P\left(\operatorname{distProb}\left(O_{i}, O_{r}\right) \leq R\right) \geq p\right\}
\end{aligned}
$$

For simplicity, from now on we will say that the uncertainty granules in $\mathcal{D}$ are the target objects (more precisely, the location granules of the target objects) and the uncertainty granule $O_{r}$ is the reference object (more precisely, the location granule of the reference object).

In the following section, we analyze this kind of constraints and present our approach to process them in an efficient way. The main problem with these queries is to calculate the probability that an object is in the answer set. Recalling the sample query in the introduction, an interesting query could be "What is the probability that a policeman is no further than 100 meters from the criminal's location?". As we will see in the next sections, the solution requires to numerically solve an integral for each of the objects in the scenario, and this calculus has a direct impact on the performance. One way to address this issue is to 
define some kind of spatial index enriched with additional information. For example, in [38], the authors define a type of $R^{*}$ tree to maintain the objects, so they can filter some objects using the $R^{*}$-tree, and the numerical integral is only calculated for a few objects. Similarly, in [45], the authors use a quadtree for the same purpose. However, in a volatile and highly-uncertain environment, where the objects are moving continuously and we cannot foresee the next movement for each object, it is difficult to maintain an index structure such an $R^{*}$-tree or a quadtree. Our goal is to obtain a method to calculate which objects are in the answer set of a probabilistic inside query without using any index structure for the objects and assuming that we have very little information about the location of the objects, that is, each object can be in any place within a disk of a given radius. However, our method is not incompatible with the use of a spatial index: It could be used jointly with an index to speed up the calculus of the query by avoiding the computation of many numerical integrals that slow down the process. In particular, as we will show in our experiments, we adopt an approach similar to [11] to show the benefits of our approach both in the presence and the absence of an index filtering the objects which are clearly out of the answer set.

\section{Processing of Probabilistic Inside Queries}

In this section, we tackle the processing of probabilistic inside queries using our model. We focus on the problem of tagging each target object with the probability that the object is part of the query answer, as applying a probability threshold specified in the constraint can be considered as a filtering step once these probabilities have been computed. For simplicity of exposition, we assume uncertainty with a continuous probability density function in all the situations and for all the objects. An analogous discussion would apply for a discrete probability mass function.

As an example of the queries to be processed, consider a query such as "Which taxis (class of target objects) are less than $R$ meters from Anne (reference object) with a probability greater than $p "$. In this case, while raw GPS locations are considered, the locations are subject to an uncertainty; therefore, the concept of uncertainty granule (see Definition 1) must be used to refer to those locations.

For illustrative purposes, let us take a look at Figure 1. In the figure, $U G_{t}$ is the uncertainty area associated to the target object $O_{t}$, and $U G_{r}$ is the uncertainty area associated to the reference object $O_{r}$; for simplicity, and without loss of generality, $U G_{r}$ is a rectangle and $U G_{r}$ is a circle. The area $A_{t}$ are the points of $U G_{t}$ which are not further than $R$ (the radius of the inside constraint) from the uncertainty area $U G_{r}$ associated to the reference object $O_{r}$. Formally:

$$
\begin{aligned}
A_{t} & =\left\{x \in U G_{t} \mid \operatorname{dist}\left(x, U G_{r}\right) \leq R\right\} \\
& =E A\left(U G_{r}\right) \cap U G_{t}
\end{aligned}
$$

where $E A\left(U G_{r}\right)$ is what we call the extended area of $U G_{r}$ (the area inside the dotted line surrounding $U G_{r}$ in Figure 1), i.e.,

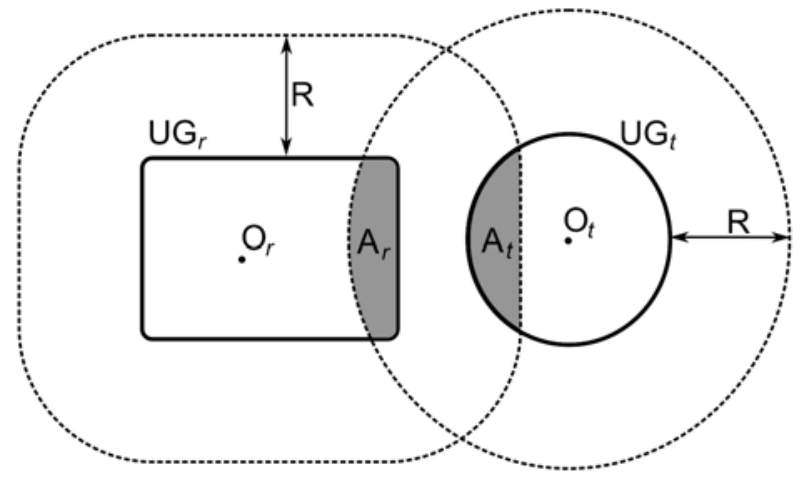

Figure 1: Areas involved in a probabilistic inside query.

the Minkowski sum of the area $U G_{r}$ and a disk with radius $R$ (the Minkowski sum of two sets in the Euclidean space is obtained by adding every element of one set to every element of the other [41]).

In the same way, the area $A_{r}$ contains the points in $U G_{r}$ that are not further than $R$ from $U G_{t}$ :

$$
\begin{aligned}
A_{r} & =\left\{x \in U G_{r} \mid \operatorname{dist}\left(x, U G_{t}\right) \leq R\right\} \\
& =E A\left(U G_{t}\right) \cap U G_{r}
\end{aligned}
$$

If we consider a may constraint (probability threshold $>0$ ), then there is no need to compute the actual probabilities of the objects (unless this information must be computed to show it to the user). In this case, a target object will be part of the answer as long as its area $A_{t}$ is not empty.

As we will see in the next subsection, calculating the exact probability that the objects $O_{t}$ and $O_{r}$ are no further than $R$ involves solving numerically a double integral that may have a great impact on the computational cost when dealing with a large number of objects, even if we assume that the $p d f$ functions of the uncertainty granules are simple. Section 4.1 is devoted to explain how objects can be sieved to decide if an object must be or cannot be in the answer set. With this sieve, we limit the number of objects for which we have to compute the exact probability.

\subsection{Calculating the Exact Probability}

This section is devoted to describe how to calculate the probability that two objects represented by the uncertainty granules $O_{1}$ and $O_{2}$ are not further than $R$, that is, how to calculate the value $P\left(\operatorname{dist} \operatorname{Prob}\left(O_{1}, O_{2}\right) \leq R\right)$ that appears in Definition 2. We first describe how to calculate the probability for the general case, i.e., making no assumption about the uncertainty area $U G_{i}$ and the density function $f_{i}$ of the uncertainty granules $O_{i}=\left\langle i d, U G_{i}, f_{i}\right\rangle$. Then, we particularize these calculations when $U G_{i}$ is a disk and $f_{i}$ is the uniform probability density function over the disk $U G_{i}$.

So, we will describe how to obtain the function

$$
\operatorname{probL}_{O_{1}}^{O_{2}}: \mathbf{R} \rightarrow[0,1]
$$


such that $\operatorname{probL}_{O_{1}}^{O_{2}}(R)=P\left(\operatorname{distProb}\left(O_{1}, O_{2}\right) \leq R\right)$. Note that $\operatorname{probL}_{\mathrm{O}_{1}}^{\mathrm{O}_{2}}$ is the cumulative distribution function of the probability density function, distProb $\left(O_{1}, O_{2}\right)$ (see Table 2 in Section 2.1).

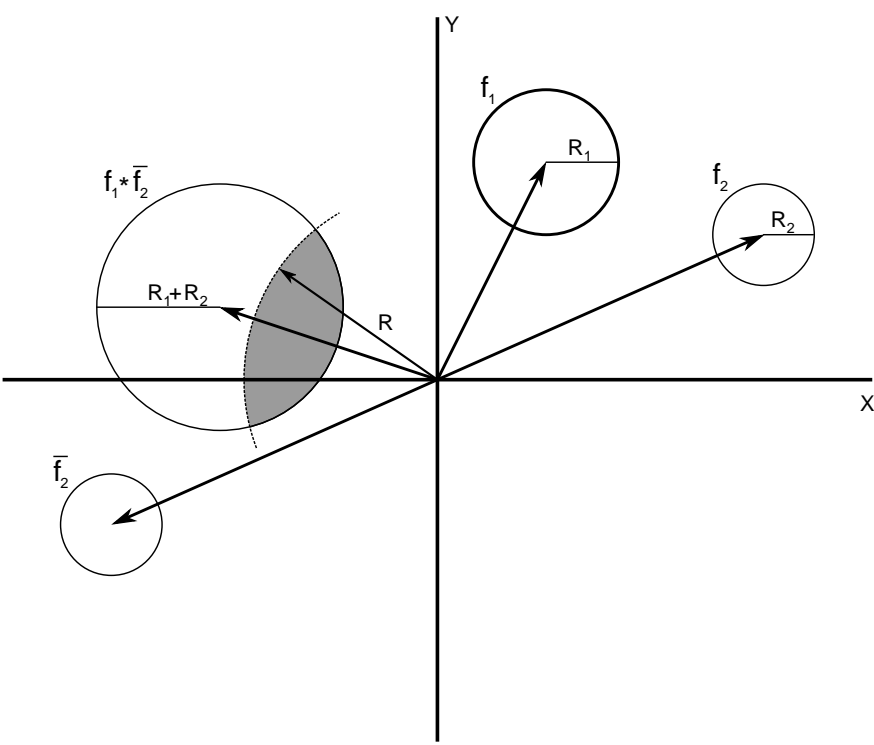

Figure 2: Probability density functions and random variables used for modeling the problem: $f_{1}, f_{2}, \bar{f}_{2}$, and $f_{1} * \bar{f}_{2}$.

Let $O_{1}$ and $O_{2}$ be two imprecise objects (uncertainty granules) $O_{1}=\left\langle i d, U G_{1}, f_{1}\right\rangle$ and $O_{2}=\left\langle i d, U G_{2}, f_{2}\right\rangle$. We will denote by $X$ the two-dimensional random variable that models where the object $O_{1}$ is situated in the plane. It is clear that $f_{1}$ is the probability density function of the random variable $X$. Similarly, we define $Y$ as a two-dimensional random variable with probability density function $f_{2}$ for $O_{2}$ (see Figure 2). Let us denote by $\bar{Y}$, the random variable, $\bar{Y}(w)=-Y(w)$ with density function $\bar{f}_{2}$, where $\bar{f}_{2}(x, y)=f_{2}(-x,-y)$ (that is, $\bar{f}_{2}$ is symmetric to $f_{2}$ with respect to the origin). This is illustrated in Figure 2.

The random variable $X+\bar{Y}$ represents all difference vectors from a possible location of $O_{1}$ to a possible location of $O_{2}$. Intuitively, the probability that the distance from $O_{1}$ to $O_{2}$ is less than $R$, i.e., $\operatorname{probL}_{O_{1}}^{O_{2}}(R)$, can be calculated dividing the number of these difference vectors whose norm is less than $R$ (all the possible locations where the objects are not further than $R$ ) by the number of all the possible difference vectors (all the possible locations of the objects). It is well known that the density function $f$ of the sum of two random variables is the convolution product (denoted by $*$ ) of the density functions of each random variable. So, the density function of $X+\bar{Y}$ is:

$$
f(\mathbf{t})=\left(f_{1} * \bar{f}_{2}\right)(\mathbf{t})=\int_{\mathbf{R}^{2}} f_{1}(\mathbf{x}) \bar{f}_{2}(\mathbf{t}-\mathbf{x}) d \mathbf{x}
$$

or equivalently:

$$
f(\mathbf{t})=\int_{\mathbf{R}^{2}} f_{1}(\mathbf{x}) f_{2}(\mathbf{x}-\mathbf{t}) d \mathbf{x}
$$

since $\bar{f}_{2}(\mathbf{t}-\mathbf{x})=f_{2}(\mathbf{x}-\mathbf{t})$.
Hence, the cumulative distribution function $\operatorname{probL}_{O_{1}}^{O_{2}}$, representing the probability that the objects are no further than $R$, is the integral of $f$ over the disk of center $(0,0)$ and radius $R$, $B(0, R)$ :

$$
\operatorname{probL}_{O_{1}}^{O_{2}}(R)=\int_{B(0, R)} f(\mathbf{t}) d \mathbf{t}=P\left(\operatorname{distProb}\left(O_{1}, O_{2}\right) \leq R\right)
$$

In general, the computation of this integral could have a great computational cost since we have to solve numerically two integrals in the plane: one integral to solve the convolution and another one to integrate this convolution over a disk.

\section{Case of Study: Uniform Pdfs}

In this section, we focus on the case of study where density functions $f_{1}$ and $f_{2}$ in Equation 1 are uniform $p d f s$ over a disk, which is a common assumption in the field of moving objects. The uniform $p d f$ is applied when there is no information about the location of an object. In that case, we consider the worst case: The object can be in any place of the uncertainty area with equal probability. Another reason to consider the uniform distribution is its simplicity and feasibility (see [11] for a extended discussion).

Thus, we will denote by $f_{1}^{R}$ the uniform probability density function over a disk centered in a location $\mathbf{l} \in \mathbf{R}^{2}$ and radius $R \in \mathbf{R}$ :

$$
f_{\mathbf{l}}^{R}(\mathbf{t})= \begin{cases}\frac{1}{\pi R^{2}}, & \mathbf{t} \in B(\mathbf{l}, R) \\ 0, & \mathbf{t} \notin B(\mathbf{l}, R)\end{cases}
$$

In the formula, $\pi R^{2}$ obviously represents the area of the disk. In this case, we can give a closed formula for the convolution of two functions, $f_{\mathbf{l}_{1}, \mathbf{l}_{2}}^{R_{1}, R_{2}}(\mathbf{t})=f_{\mathbf{l}_{1}}^{R_{1}} * \bar{f}_{\mathbf{l}_{2}}^{R_{2}}(\mathbf{t})$, doing some geometrical calculations:

$$
f_{\mathbf{l}_{1}, \mathbf{l}_{2}}^{R_{1}, R_{2}}(\mathbf{t})= \begin{cases}\frac{1}{\pi^{2} R_{1}^{2} R_{2}^{2}} A(\|\mathbf{t}\|) & \mathbf{t} \in B\left(\mathbf{l}_{1}-\mathbf{l}_{2}, R_{1}+R_{2}\right) \\ 0 & \text { otherwise }\end{cases}
$$

where $A(\| \mathbf{t} \mid)$ is the area of $B\left((0,0), R_{2}\right) \cap B\left((\|\mathbf{t}\|, 0), R_{1}\right)$ and $\|\mathbf{t}\|$ represents the norm of the vector $\mathbf{t}$, as shown in Figure 3.

If we calculate the area $A(\|\mathbf{t}\|)$, the function $f_{\mathbf{l}_{1}, \mathbf{l}_{2}}^{R_{1}, R_{2}}(\mathbf{t})$ is equal to:

$$
\begin{cases}\frac{1}{\pi \max \left(R_{1}^{2}, R_{2}^{2}\right)}, & || \mathbf{l}_{1}-\mathbf{l}_{2}-\mathbf{t} \| \leq\left|R_{1}-R_{2}\right| \\ \frac{F_{R_{1}, R_{2}}(\| \mathbf{t} \mid)}{\pi^{2} R_{1}^{2} R_{2}^{2}}, & \left|R_{1}-R_{2}\right| \leq\left\|\mathbf{l}_{1}-\mathbf{l}_{2}-\mathbf{t}\right\| \leq R_{1}+R_{2} \\ 0, & \text { otherwise }\end{cases}
$$

where $F_{R_{1}, R_{2}}(x)=A_{R_{1}}^{R_{2}}(x)+A_{R_{2}}^{R_{1}}(x)-T_{R_{1}, R_{2}}(x) / 2$, and:

$$
\begin{aligned}
A_{k_{1}}^{k_{2}}(x) & =k_{1}^{2} \arccos \left(\frac{k_{1}^{2}-k_{2}^{2}+x^{2}}{2 k_{1} x}\right) \\
T_{R_{1}, R_{2}}(x) & =\sqrt{4 R_{1}^{2} R_{2}^{2}-\left(R_{1}^{2}+R_{2}^{2}-x^{2}\right)^{2}}
\end{aligned}
$$




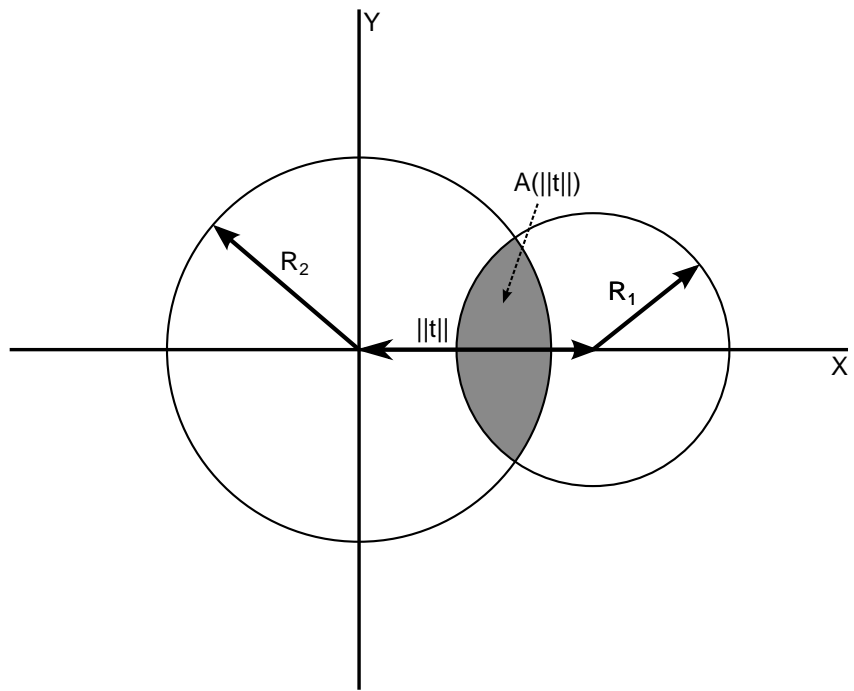

Figure 3: Inside constraint: $A(\|\mathbf{t}\|)$ for the uniform probability density function case.

In Figure 4 , the shape of a function $f_{\mathbf{l}_{1}, \mathbf{l}_{2}}^{R_{1}, R_{2}}(\mathbf{t})$ can be seen. The top circle has radius $\left|R_{1}-R_{2}\right|$, the bottom one has radius $R_{1}+R_{2}$, and its center is situated in $\mathbf{l}_{1}-\mathbf{l}_{2}$.

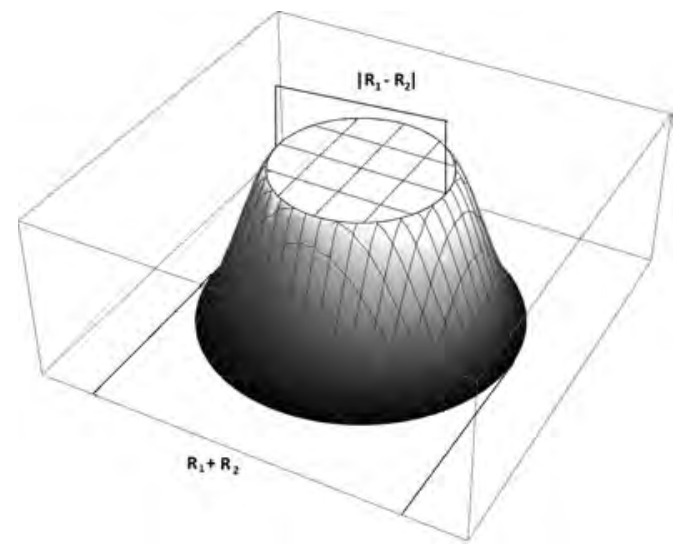

Figure 4: Shape of the function $f_{\mathbf{l}_{1}, \mathbf{l}_{2}}^{R_{1}, R_{2}}(t)$ for the uniform probability density function case. The center of the bottom circle is at $\mathbf{l}_{1}-\mathbf{l}_{2}$.

It is easy to see that Figure 4 is the solid of revolution generated by the curve (see Figure 5) $g: \mathbf{R} \rightarrow \mathbf{R}$, defined as:

$$
g(x)= \begin{cases}\frac{1}{\pi R_{2}^{2}}, & 0 \leq x \leq R_{2}-R_{1} \\ \frac{1}{\pi^{2} R_{1}^{2} R_{2}^{2}} F_{R_{1}, R_{2}}(x), & R_{2}-R_{1} \leq x \leq R_{2}+R_{1} \\ 0, & \text { otherwise }\end{cases}
$$

Therefore, the probability that the distance between objects $O_{1}$ and $O_{2}$ with associated density functions $f_{\mathbf{l}_{1}}^{R_{1}}$ and $f_{\mathbf{l}_{2}}^{R_{2}}$ is less than $R$ can be computed by:

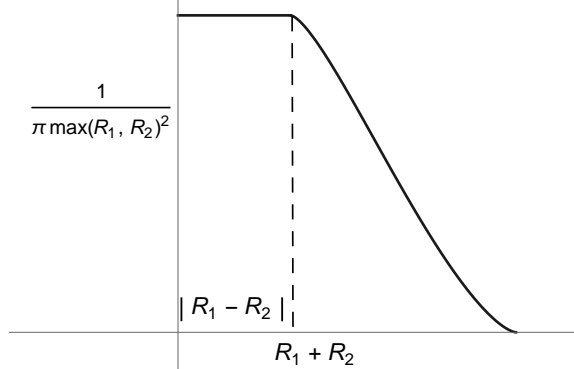

Figure 5: Curve that generates the function $f_{\mathbf{l}_{1}, \mathbf{l}_{2}}^{R_{1}, R_{2}}$ for the uniform probability density function case.

$$
\operatorname{probL}_{O_{1}}^{O_{2}}(R)=\int_{B(0, R)} f_{\mathbf{l}_{1}, \mathbf{l}_{2}}^{R_{1}, R_{2}}(\mathbf{t}) d \mathbf{t}=2 \pi \int_{0}^{R} x g(x) d x
$$

Due to the symmetry of the problem, the following proposition can be proved, that will be used to simplify the computation of $\operatorname{probL}_{O_{1}}^{O_{2}}(R)$ :

Proposition 1. Let $f_{\mathbf{l}_{1}}^{R_{1}}$ and $f_{\mathbf{l}_{2}}^{R_{2}}$ be uniform density functions over the disks of center $\mathbf{l}_{1}$ and $\mathbf{l}_{2}$, and radius $R_{1}$ and $R_{2}$, respectively. If we denote by $d_{0}$ the distance from $\mathbf{l}_{1}$ to $\mathbf{l}_{2}, d_{0}=\left\|\mathbf{l}_{1}-\mathbf{l}_{2}\right\|$, then:

$$
\int_{B(0, R)} f_{\mathbf{l}_{1}, \mathbf{l}_{2}}^{R_{1}, R_{2}}(\mathbf{t}) d \mathbf{t}=\int_{B(0, R)} f_{(0,0),\left(d_{0}, 0\right)}^{R_{1}, R_{2}}(\mathbf{t}) d \mathbf{t}
$$

Hence, the value of $\operatorname{probL}_{\mathrm{O}_{1}}^{\mathrm{O}_{2}}(R)$ for uncertainty granules with uniform probability density functions over disks depends only on the distance between the centers of the disks.

A geometrical interpretation of the value $\operatorname{probL}_{O_{1}}^{O_{2}}(R)$ is shown in Figure 6: $\operatorname{probL}_{O_{1}}^{O_{2}}(R)$ is the volume over the circumference of radius $R$ and the function $f_{\mathbf{1}_{1}, \mathbf{l}_{2}}^{R_{1}, R_{2}}$.

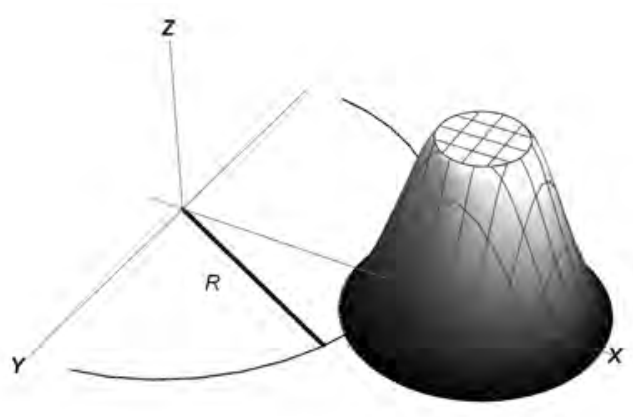

Figure 6: Interpretation of $\operatorname{probL}_{O_{1}}^{O_{2}}(R)$ for uncertainty granules with uniform probability density functions.

It is not possible to give a closed formula to compute the value of $\operatorname{probL}_{O_{1}}^{O_{2}}(R)$ when $O_{i}$ has an arbitrary uniform $p d f f_{\mathbf{l}_{i}}^{R_{i}}$, so it could be necessary to compute it numerically. That has a 
great impact on the performance, as we will see in Section 5. To avoid this problem, we will sieve the objects that must be or cannot be in the answer set, so the integral will be computed for a small set of objects. In the next section, we will explain how to perform this sifting. We will see that we can find a closed formula to compute probL $\mathrm{O}_{\mathrm{O}_{1}}(R)$ if $\mathbf{l}_{1}=\mathbf{l}_{2}$ in Equation (4) (and so, in that case, we do not need to compute it numerically). Moreover, we will explain how we can obtain an upper and a lower bound for $\operatorname{probL}_{O_{1}}^{O_{2}}(R)$ when $\mathbf{l}_{1} \neq \mathbf{l}_{2}$, which allow us to sieve many of the candidates with less effort.

\subsection{Optimization when a Minimum Probability is Required: Probability Threshold}

As we mentioned in the previous section, the integral of Equation (4) has to be solved numerically. In a scenario with hundreds of objects, if we want to retrieve the objects that are not further than $R$ from a reference object with a given probability (probability threshold), the integral has to be calculated numerically hundreds of times, which is computationally expensive. In this section, we will describe how to sift out the objects to avoid calculating the integral as many times as possible. We start with some notations that we will use in the rest of the paper.

Definition 3. We denote by $C_{1}^{d_{1}, d_{2}}$ the annulus formed by two circles centered at $\mathbf{1}$ and radius $d_{1}$ and $d_{2}\left(d_{1}<d_{2}\right.$, see Figure 7.a) :

$$
C_{\mathbf{l}}^{d_{1}, d_{2}}=B\left(\mathbf{l}, d_{2}\right) \backslash B\left(\mathbf{l}, d_{1}\right)
$$

We also denote by $C_{1}^{d_{1}, d_{2}}\left(\theta_{1}, \theta_{2}\right)$ the portion of the annulus $C_{1}^{d_{1}, d_{2}}$ between the lines passing through $\mathbf{I}$ with slopes equal to $\theta_{1}$ and $\theta_{2}$, that is (see Figure 7.b):

$$
C_{\mathbf{l}}^{d_{1}, d_{2}}\left(\theta_{1}, \theta_{2}\right)=\left\{\mathbf{y} \in C_{\mathbf{1}}^{d_{1}, d_{2}} \mid \theta_{1} \leq \widehat{\mathbf{y}-\mathbf{l}} \leq \theta_{2}\right\}
$$

where $\widehat{\mathbf{y}-1}$ is the angle between $\mathbf{y}-\mathbf{l}$ and the $x$-axis.

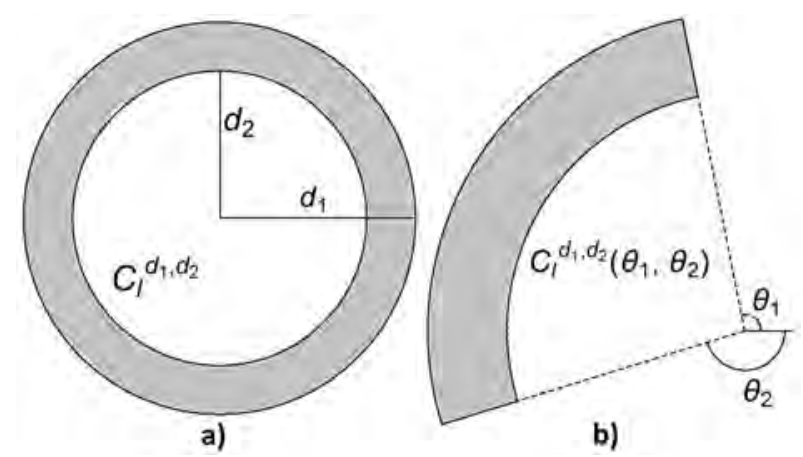

Figure 7: Geometric interpretation of an annulus, $C_{1}^{d_{1}, d_{2}}$ (a); and a portion of an annulus, $C_{1}^{d_{1}, d_{2}}\left(\theta_{1}, \theta_{2}\right)(\mathrm{b})$.

Definition 4. We will denote by $B_{R_{1}, R_{2}}(x)$ the value of the integral:

$$
B_{R_{1}, R_{2}}(x)=\int_{B(0, x)} f_{\mathbf{l}_{0}, \mathbf{l}_{0}}^{R_{1}, R_{2}}(\mathbf{t}) d \mathbf{t}
$$

Note that $B_{R_{1}, R_{2}}(x)$ does not depend on the choice of $\mathbf{l}_{0}$ by Proposition 1. Geometrically, the value $B_{R_{1}, R_{2}}(x)$ is the volume of the intersection of a cylinder of radius $x$ centered on the coordinate origin and $f_{\mathbf{l}_{0}, \mathbf{l}_{0}}^{R_{1}, R_{2}}$.

Using the notation of Equations (2) and (3), it can be shown that $B_{R_{1}, R_{2}}(x)$ is equal to:

$$
\left\{\begin{array}{lc}
\frac{x^{2}}{\max \left(R_{1}^{2}, R_{2}^{2}\right)}, & 0 \leq x \leq\left|R_{1}-R_{2}\right| \\
\frac{1}{\pi R_{1}^{2} R_{2}^{2}}\left[x^{2}\left(A_{R_{1}}^{R_{2}}(x)+A_{R_{2}}^{R_{1}}(x)\right)+R_{2}^{2} A_{R_{1}}^{x}\left(R_{2}\right)\right. \\
\left.-1 / 4\left(R_{1}^{2}+R_{2}^{2}+x^{2}\right) T_{R_{1}, R_{2}}(x)\right], \\
\\
\left|R_{1}-R_{2}\right|<x<R_{1}+R_{2} \\
1, \quad x \geq R_{1}+R_{2}
\end{array}\right.
$$

The basic idea of the sifting process is that $B_{R_{1}, R_{2}}(x)$ can be calculated using the closed formula (5).

Let $S=\left\{O_{t_{i}} \mid 1 \leq t \leq m\right\}$ be a set of target objects and $O_{r}$ be a reference object. The process of sifting the set $S$ is based on obtaining a lower and an upper bound for the value of the integral corresponding to $\operatorname{probL}_{O_{r}}^{O_{t_{i}}}(R)$. If we want to retrieve the objects of $S$ that are not further than $R$ from $O_{r}$ with a probability greater than $p$, and it is known that $\operatorname{probL}_{O_{r}}^{O_{t_{i}}}(R) \in\left[l_{i}, u_{i}\right]$, then the objects such that $p<l_{i}$ are in the answer set and the objects with $u_{i}<p$ are not in the answer set. So, we have to calculate the integral only for the objects where $p \in\left[l_{i}, u_{i}\right]$.

Now, we will explain in detail how to get the lower and the upper bound, $[l, u]$, for $\operatorname{probL}_{O_{r}}^{O_{t}}(R)$, where $O_{r}$ and $O_{t}$ are the uncertainty granules with associated density functions $f_{\mathbf{l}_{1}}^{R_{1}}$ and $f_{\mathbf{l}_{2}}^{R_{2}}$, respectively. By Proposition 1 , we can assume that $\mathbf{l}_{1}=(0,0)$ and $\mathbf{l}_{2}=\left(d_{0}, 0\right)$, where $d_{0}$ is the distance from $\mathbf{l}_{1}$ to $\mathbf{l}_{2}$. We want to give an upper and a lower bound of the volume of $f_{\mathbf{l}_{1}, \mathbf{l}_{2}}^{R_{1}, R_{2}}$ under the area $A=B((0,0), R) \cap B\left(\left(d_{0}, 0\right), R_{1}+R_{2}\right)$ (gray area in Figure 8):

$$
\operatorname{probL}_{O_{r}}^{O_{t}}(R)=\int_{A} f_{\mathbf{l}_{1}, \mathbf{l}_{2}}^{R_{1}, R_{2}}
$$

Let us denote by $\left[m_{l}, m_{u}\right]$ the interval that is the intersection of $A$ and the $x$-axis. We divide the interval $\left[d_{0}-m_{u}, d_{0}-m_{l}\right]$ in $n$ equal parts:

$$
\left[d_{0}-m_{u}, d_{0}-m_{l}\right]=\cup_{i=1}^{n}\left[x_{i}, x_{i+1}\right]
$$

where obviously $x_{1}=d_{0}-m_{u}$ and $x_{n+1}=d_{0}-m_{l}$. If we denote by $C_{i}=C_{\left(d_{0}, 0\right)}^{x_{i}, x_{i+1}}$ the annulus centered at $\left(d_{0}, 0\right)$ and radius $x_{i}$ and $x_{i+1}$, we can split the area $A$ into $n$ parts, $A=A_{1} \cup \cdots \cup A_{n}$ with $A_{i}=C_{i} \cap A$ (see Figure 8).

Let $\alpha_{i}$ be the maximum angle such that $L_{i}=C_{\left(d_{0}, 0\right)}^{x_{i}, x_{i+1}}\left(\alpha_{i},-\alpha_{i}\right)$ holds that $L_{i} \subseteq A_{i}$ (see Figure 9.a), and $\beta_{i}$ be the minimum angle such that $A_{i} \subseteq U_{i}=C_{\left(d_{0}, 0\right)}^{x_{i}, x_{i+1}}\left(\beta_{i},-\beta_{i}\right)$ (see Figure 9.b). It is clear that $L=\cup_{i=1}^{n} L_{i} \subseteq A \subseteq U \stackrel{d^{\prime}}{=} \cup_{i=1}^{n} U_{i}$. Therefore:

$$
l=\int_{L} f_{\mathbf{l}_{1}, \mathbf{l}_{2}}^{R_{1}, R_{2}} \leq \int_{A} f_{\mathbf{l}_{1}, \mathbf{l}_{2}}^{R_{1}, R_{2}} \leq \int_{U} f_{\mathbf{l}_{1}, \mathbf{l}_{2}}^{R_{1}, R_{2}}=u
$$




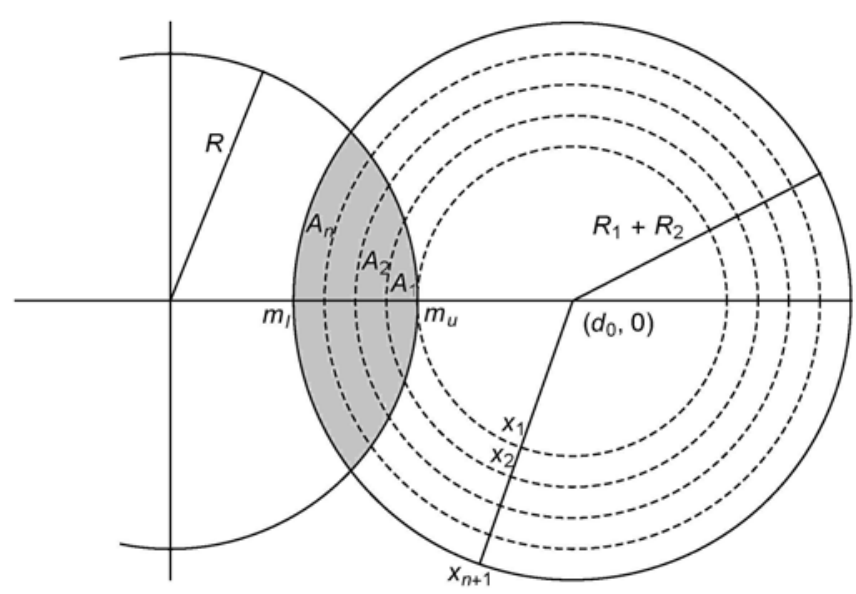

Figure 8: Annuli used to give a lower and upper bound for the sieve.
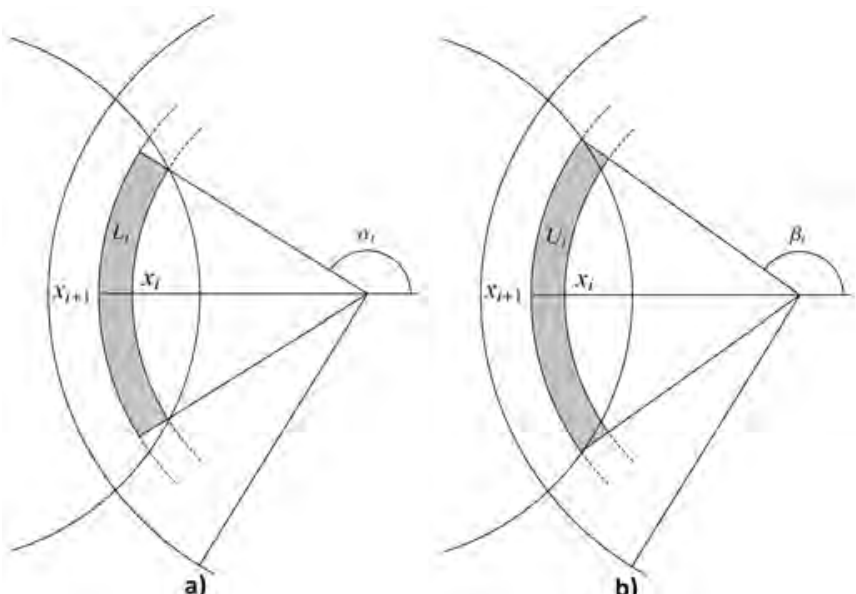

b)

Figure 9: Minimum bound for $A_{i}$ (a); and maximum bound for $A_{i}$ (b).

Hence, $l$ and $u$ are bounds satisfying that $\operatorname{probL}_{O_{r}}^{O_{t}} \in[l, u]$.

Now, let us note that the value $l$ can be calculated using $B_{R_{1}, R_{2}}(x)$ (see Definition 4):

$$
\begin{aligned}
l & =\int_{L} f_{\mathbf{l}_{1}, \mathbf{l}_{2}}^{R_{1}, R_{2}}=\sum_{i=1}^{n} \int_{L_{i}} f_{\mathbf{l}_{1}, \mathbf{l}_{2}}^{R_{1}, R_{2}}= \\
& =\sum_{i=1}^{n} \frac{\left(\pi-\alpha_{i}\right)}{\pi}\left(B_{R_{1}, R_{2}}\left(x_{i+1}\right)-B_{R_{1}, R_{2}}\left(x_{i}\right)\right)= \\
& =\sum_{i=1}^{n+1} \frac{M_{i}}{\pi} B_{R_{1}, R_{2}}\left(x_{i}\right)
\end{aligned}
$$

where $M_{i}=\pi-\alpha_{i}$ if $i=1$ or $i=n+1$, and $M_{i}=\alpha_{i}-\alpha_{i-1}$ if $i=2, \ldots, n$. In a similar way, we can deduce that the upper bound $u$ is equal to:

$$
u=\sum_{i=1}^{n+1} \frac{N_{i}}{\pi} B_{R_{1}, R_{2}}\left(x_{i}\right)
$$

where $N_{i}=\pi-\beta_{i}$ if $i=1$ or $i=n+1$, and $N_{i}=\beta_{i}-\beta_{i-1}$ if $i=2, \ldots, n$.

Finally, the angles $\alpha_{i}$ and $\beta_{i}$ can be also computed with a closed formula since, basically, their computation is equivalent to finding the point where $B((0,0), R)$ and $B\left(\left(d_{0}, 0\right), x_{i}\right)$ intersect (see the notation of Equation 2):

$$
\begin{aligned}
\alpha_{i} & =A_{R_{1}+R_{2}}^{R}\left(x_{i}\right) /\left(R_{1}+R_{2}\right)^{2} \\
\beta_{i} & =A_{R_{1}+R_{2}}^{R}\left(x_{i+1}\right) /\left(R_{1}+R_{2}\right)^{2}
\end{aligned}
$$

Therefore, we can quickly calculate a lower and an upper bound for $\operatorname{probL}_{O_{r}}^{O_{t}}$, since we have a closed formula. In Section 5, we will see empirically the benefits of these bounds.

\section{Experimental Evaluation}

To validate our proposal in terms of performance and scalability, we have performed an extensive experimental evaluation. In this section, we first specify the implementation details of our prototype and the experiment settings (Section 5.1). Then, we present some experimental results in two different sections: Section 5.2 is dedicated to tests focused on measuring the filtering capabilities of our approach, while Section 5.3 is dedicated to tests focusing on evaluating the performance over both synthetic and real datasets.

\subsection{Experimental Settings}

We have developed a prototype to show the feasibility of our approach, capable of processing probabilistic location-based queries in the presence of location uncertainty. Our prototype has been implemented using Java 1.7 as the programming language, which has allowed us to test it on Desktop PCs and Android devices (see specifications later).

In the experiments, we have used both synthetic and real datasets. We have generated five sets of 1,000,000 objects each, which are randomly placed in a 4000x4000 grid, as synthetic datasets, which we have made available at http://sid.cps. unizar.es/projects/ProbabilisticQueries/datasets/. As real dataset, we have used the LB dataset, a dataset containing the minimum bounding rectangles (MBRs) of Long Beach county roads. It contains $53 \mathrm{~K}$ pairs of points, but we have considered each of the points in the tuples as a possible location for an object, leading to a $106 \mathrm{~K}$ point dataset. This dataset has been produced by the Tiger project of the US Census Bureau, and it is downloadable at the R-tree portal, http: //www.rtreeportal.org.

We focus on evaluating the query processing where a minimum probability threshold has been established in the query. We take as ground-truth probability values the ones obtained using the Simpson's rule ${ }^{2}$ in $2 \mathrm{D}$ for integration to calculate the integrals using 100 steps for each dimension. Moreover, we

\footnotetext{
${ }^{2}$ Simpson's rule is a well known integration method. A brief introduction to this method can be found in http://en.wikipedia.org/wiki/Simpson' s_rule or in http://spikedmath.com/336.html.
} 
consider that the uncertainty area of all the reference and the target objects is a disk and the $p d f$ associated is the uniform distribution over the disk ${ }^{3}$. The specific uncertainty and query range radii varies from experiment to experiment depending on the particular aspect of our approach we are evaluating, and it will be specified at the beginning of each of the subsequent experiment sections.

In order to effectively assess the benefits of our approach in presence of possible spatial indexing techniques, our prototype is able to use a filter based on a PR-tree [2] to index and prefilter the objects in the scenario using the minimum bounding boxes (MBBs) of the uncertainty areas of the objects ${ }^{4}$. The main idea behind this prefiltering step is that whenever the MBB of an object does not overlap the MBB of the uncertainty area of the reference object extended by the range radius of the query, the probability that such an object belongs to the answer set is 0 , and can be directly discarded without performing further calculations. Thus, when this index-based filter is used, our sieve is applied to the set of objects that are not pruned by it. In our experiments, whenever this index-based filter is used, it will be explicitly stated; otherwise, the evaluation is performed over all the objects in the scenario without using any index.

Finally, the experiments were run on a computer with an Intel Core i5-2320 processor running at $3.00 \mathrm{GHz}$ and $16 \mathrm{~GB}$ of RAM memory, and on two different Android smartphones, a Galaxy Nexus (Android 4.2.1, Texas Instruments OMAP 4460 dual-core 1.2 GHz, 1 GB RAM, released in 2011), and a BQ M5 (Android 5.1.1, Qualcomm Snapdragon 615 octa-core $1.5 \mathrm{GHz}$, 2 GB RAM, released in 2015).

\subsection{Evaluating Filtering Capabilities}

The first set of tests was focused on evaluating the filtering capabilities of the method presented in Section 4.1, that is, the percentage of objects that are correctly evaluated to be part or not of the answer without having to calculate the exact probability. The filtering efficiency was measured in two different settings: with no previous object indexing (i.e., the calculations are performed against all the objects in the scenario, regardless their position), and using a PR-tree to prefilter all the objects that could never be part of the answer set. This latter prefiltering is performed taking into account the minimum bounding box of the uncertainty area of each object in the scenario, and the minimum bounding box of the query relevance area (defined by the uncertainty radius plus the range radius): if such minimum bounding boxes do not overlap the object is discarded, as the probability that it belongs to the answer set is 0 .

In these experiments, we set high levels of uncertainty (uncertainty radius range 50-500 distance units), along with large inside radius (500-1500 distance units, which is from $12.5 \%$ to $37.5 \%$ of the length of the scenario) to involve as many objects as possible. Thus, we evaluate the performance in difficult

\footnotetext{
${ }^{3}$ Similar to the error given by a GPS position, assuming no further information on the probabilities.

${ }^{4}$ We have used the implementation by Robert Olofsson, which can be obtained at http://www.khelekore.org/prtree/, last accessed 29th December, 2015.
}

scenarios. The values that we have considered for the parameters in a query $q\left(\mathcal{D}, O_{r}, R, p\right)$, as described in Definition 2, can be seen in Table 3. We have run queries for five locations of the reference object in the 4000x4000 grid, and all the possible combinations of the other parameters.

Table 3: Parameters for the filtering experiments of a query $q\left(\mathcal{D}, O_{r}, R, p\right)$.

\begin{tabular}{|l|l|}
\hline Total target objects in $\mathcal{D}$ & $10^{2}, 10^{3}, 10^{4}, 10^{5}$ \\
\hline $\begin{array}{l}\text { Locations of the reference ob- } \\
\text { ject }\left(O_{r}\right)\end{array}$ & $\begin{array}{l}(2000, \\
(2000 \pm 1500, \\
2000 \pm 1500)\end{array}$ \\
\hline $\begin{array}{l}\text { Radius for uncertainty area of } \\
\text { reference and target objects }\end{array}$ & $50,100,250,500$ \\
\hline$R$ (inside radius) & $500,1000,1500$ \\
\hline$p$ (probability threshold) & $0.25,0.50,0.751 .0$ \\
\hline Number of annuli & $16,32,64$ \\
\hline
\end{tabular}

The results of the first filtering experiment (no preindexing) are shown in Figure 10, which displays the mean of the filtering rate when 16,32 , and 64 annuli are used to obtain the filtering bounds. For instance, the mean of the filtering rate for all the possible queries combining the parameters of Table 3 with 100 objects and using 64 annuli is $99.92 \%$. We can observe that the numerical integral to obtain the exact probability has to be calculated for less than $1 \%$ of the target objects.

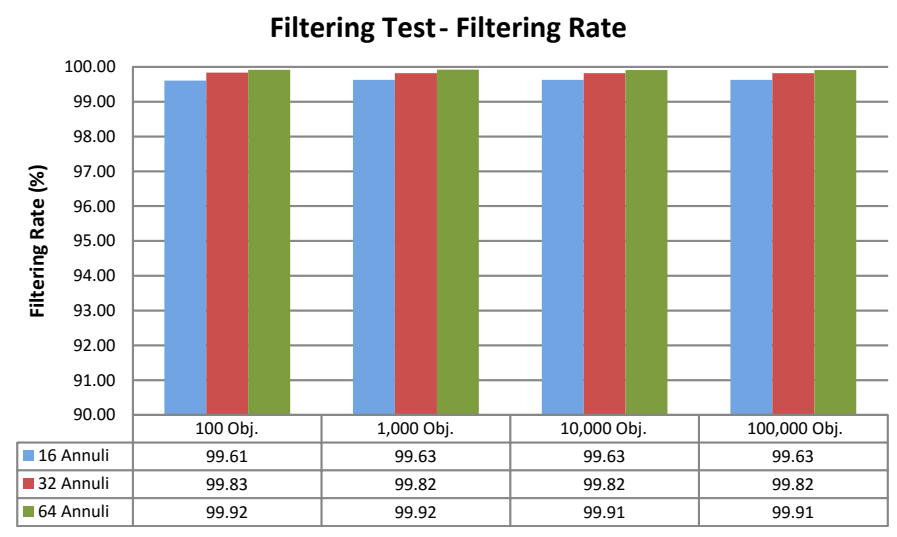

Figure 10: Filtering rate varying the number of annuli without indexing.

In Figure 11, the results of applying our approach after prefiltering the objects using a PR-tree based filter are shown. The graph should be read as follows:

- The first column of each group displays the mean percentage of target objects that the PR-tree based filter has been able to filter out. For example, for 100,000 objects, it was able to detect and remove $44.86 \%$ of the objects that were not going to be part of the answer set (probability 0 ) thanks to the use of the index.

- Each of the associated columns is the mean of the filtering rate of our approach over the results of the prefiltering step. Following with the previous example, for 100,000 objects and using 32 annuli, a $55.14 \%$ of the objects in 
the scenario were passed to our filtering approach, which was able to successfully determine whether the objects belonged or not to the answer set while avoiding the calculation of the integral in $99.32 \%$ of the cases.

\section{Filtering Test - Filtering Rate After PR-tree}

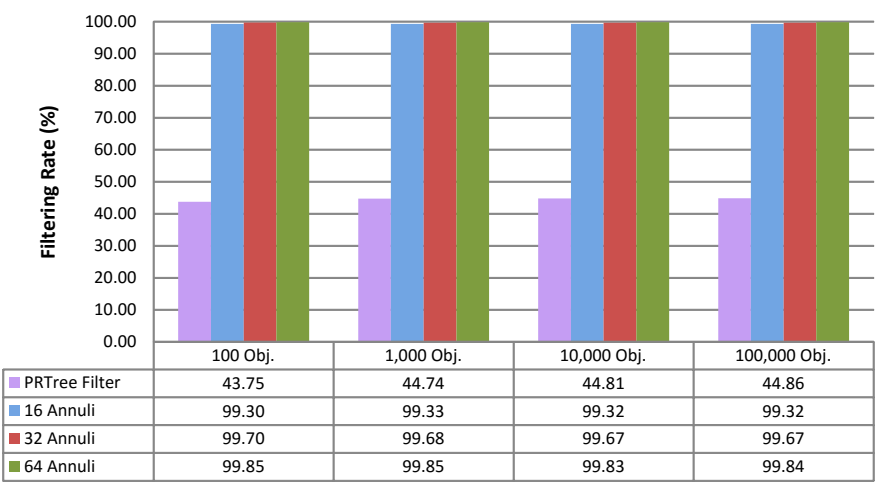

Figure 11: Filtering rate using the PR-tree-based filter and varying the number of annuli.

In all the filtering experiments, we can see how the amount of annuli used in the approximation has a direct impact on the filtering rate. However, there is a balance between the precision of the approximation and the speedup obtained by the filtering, as we will see in the following section. For completeness's sake, we include in Figure 12 the performance graph of these experiments, which displays the mean execution times grouped by the number of objects in the scenario. There, the performance of the base approach using indexes (i.e., a PR-tree based approach without our sieve step) is compared to our approach without using an index (i.e., calculating the query against all the objects in the scenario).

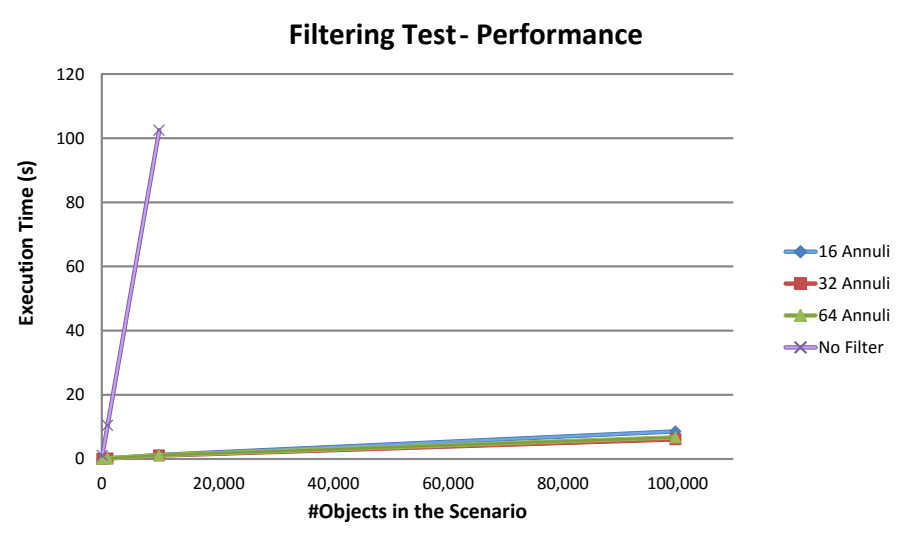

Figure 12: Performance of our approach in the filtering tests.

\subsection{Evaluating the Performance and Scalability}

The second set of tests was focused on testing the performance and scalability of our algorithm using both synthetic and real datasets.

\section{Using Synthetic Datasets}

In this set of experiments, we reduced the uncertainty (the uncertainty radius ranged from 10 to 50 units) and the radius for the range queries (from 100 to 500 units), but we increased the number of objects in the scenario up to $1,000,000$ objects (see Table 4).

Table 4: Parameters for the performance experiments of a query $q\left(\mathcal{D}, O_{r}, R, p\right)$.

\begin{tabular}{|l|l|}
\hline Total target objects in $\mathcal{D}$ & $\begin{array}{l}5 \times 10^{4}, 10^{5}, 5 \times 10^{5}, \\
10^{6}\end{array}$ \\
\hline Locations object reference $\left(O_{r}\right)$ & $\begin{array}{l}(2000, \\
(2000 \pm 1500, \\
2000 \pm 1500)\end{array}$ \\
\hline $\begin{array}{l}\text { Radius for uncertainty area of } \\
\text { reference and target objects }\end{array}$ & $10,30,50$ \\
\hline$R$ (inside radius) & $100,300,500$ \\
\hline$p$ (probability threshold) & $0.25,0.50,0.75$ \\
\hline Number of annuli & $16,32,64$ \\
\hline
\end{tabular}

We measured the performance both not using the indexbased filtering and using it, whose mean results grouped by the number of objects in the scenario can be seen in Figures 13 and 14, respectively. In the graphs, we can see the balance between the precision of the approximation (number of annuli) and the performance achieved. As the number of annuli increases, our approximation is more expensive in terms of execution time and, although it is linear in the number of objects in the scenario, such cost becomes more significant than the cost of calculating the avoided numeric integrals ${ }^{5}$.

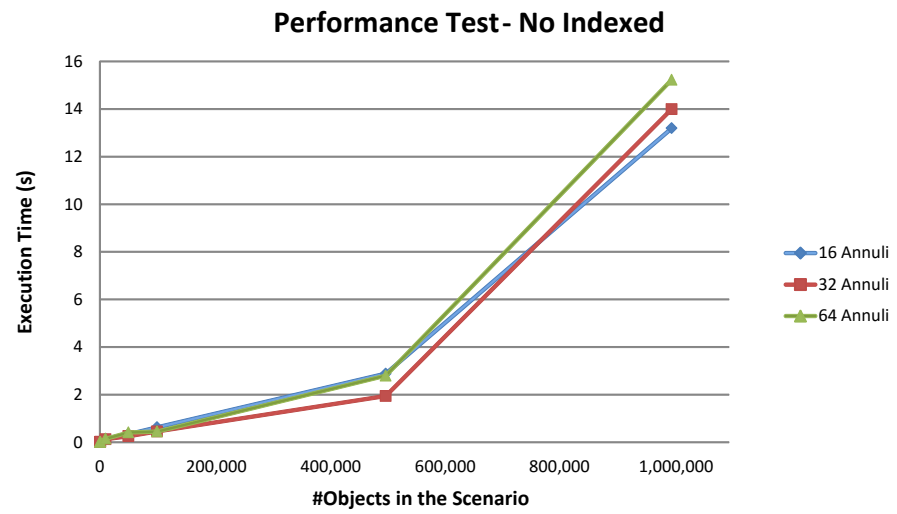

Figure 13: Performance and influence of the number of annuli in our approach when no index is used.

This can be seen in both figures, where the times spent for the tests with 64 annuli are slightly above the times spent for the tests with 32 annuli. Moreover, in Figure 13, note how for $1,000,000$ objects, the times for the tests with 32 annuli are no longer below the performance for 16 annuli due to this balance. This does not happen for the indexed tests as, thanks to the use

\footnotetext{
${ }^{5}$ This depends directly on the numeric method used to calculate the integrals. In our case, the Simpson's algorithm is quadratic in the number of steps.
} 


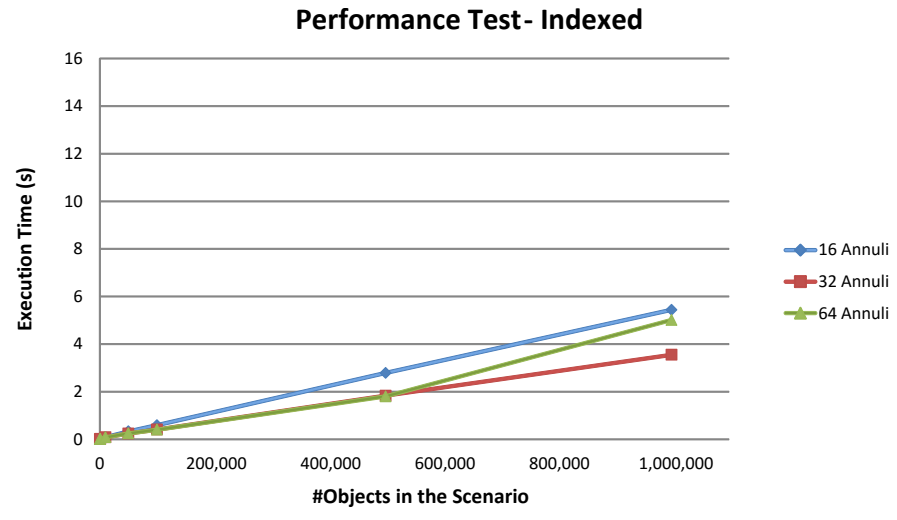

Figure 14: Performance and influence of the number of annuli in our approach using a PR-tree based indexing.

of the PR-tree, our approach is not applied to all the objects in the scenario.

Needless to say, if we did not use the filter bounds, the execution time would be huge, which clearly shows the interest and the benefits of the proposed filtering technique.

\section{Using a Real Dataset}

In order to evaluate our approach in a real scenario, we conducted a set of performance experiments on the LB dataset (described in Section 5.1). To do so, we took the same parameters used in [38] with the difference that we forced the reference object of the query to follow a trajectory of 25 steps which crossed the cloud of points of the dataset (selecting the position of the reference object randomly led to many queries with no objects in the answer and biased the results, so we chose a more challenging scenario). The parameters used for these tests can be found in Table 5. Based on the results shown in the previous set of experiments, we set the number of annuli to 32 as it is the optimal value to achieve the best performance in a scenario with such number of objects. Besides testing our approach with a real spatial dataset, we also wanted to evaluate our approach on mobile devices to determine whether it could be used in devices with limited capabilities. Thus, we implemented an Android version of the tests, and ran it on two different devices (described in Section 5.1).

Table 5: Parameters for the LB dataset experiments of a query $q\left(\mathcal{D}, O_{r}, R, p\right)$.

\begin{tabular}{|l|l|}
\hline Total target objects in $\mathcal{D}$ & $106 \times 10^{3}$ \\
\hline $\begin{array}{l}\text { Locations of the reference ob- } \\
\text { ject }\left(O_{r}\right)\end{array}$ & $\begin{array}{l}25 \text { steps within the LB } \\
\text { cloud of points }\end{array}$ \\
\hline $\begin{array}{l}\text { Radius for uncertainty area of } \\
\text { reference and target objects }\end{array}$ & $5,100,250$ \\
\hline$R$ (inside radius) & $250,500,750$ \\
\hline$p$ (probability threshold) & $0.25,0.50,0.75$ \\
\hline Number of annuli & 32 \\
\hline
\end{tabular}

The filtering rates and performance results of these tests can be seen in Figures 15 and 16, respectively. There, the results are grouped by uncertainty radius, e.g., the label LB-5 repre- sents the aggregated results of the tests using the scenario LB with an uncertainty radius of 5 distance units.

In Figure 15, we can see how the filtering rates achieved by our approach are consistent with those achieved in the synthetic scenarios. The figures should be read as follows. PRTree Prefilter is the mean filtering rate achieved by the PRTree prefilter, Filter is the mean filtering rate of our approach when applied to the objects that the prefiltering step has not discarded (similar to Figure 11) and, finally, Total is the global mean filtering of our proposal independently of using the index-based prefilter or not (similar to Figure 10). Note how the efficiency of the PRTree-based prefilter decreases as the uncertainty and query radii increase. This is due to the fact that the difference between the area of relevance (i.e., the area where the objects must lay to be part of the answer set) and its minimum bounding box increases as the sum of the uncertainty radius and the inside range radius grows, leading to more false positives (i.e., objects whose probability of being part of the answer set is 0 but are not discarded in the prefiltering step using the PRTree); however, all of those false positives are quickly removed by our sieve. Of course, these filtering rates are exactly the same for both PC and Android devices.

Regarding the performance, we can see the benefits of using our approach even not having indexed the objects in the scenario, as the calculations needed to sieve the results are faster than calculating their exact probabilities. Besides, the results obtained for Android devices suggest that this technique could be carried out by the mobile devices, without having to rely on a back-end server to perform the calculations.

\section{Related Work}

Inside queries $[8,17]$ have been studied in the literature of spatio-temporal and moving object databases (see [23] for a survey). However, existing works on location-dependent query processing usually implicitly assume GPS locations for the objects in a scenario. Although some proposals acknowledge the importance of considering different location resolutions (e.g., [19]), the processing of classical constraints such as inside is not considered in that context.

The importance of dealing with the uncertainty of location information is emphasized in the literature. Probabilistic queries, even though not in the context of moving objects, were introduced in [10]. For moving objects, probabilistic queries are usually computed by estimating the locations of the objects through a probability density function that models the uncertainty, in a way that the probability that an object is within a certain region can be computed by integration (see [11]). As solving these integrals is frequently expensive (numerical methods are usually required), an index-based filtering step is introduced to prune the search space. Different relevant proposals exist in the literature. For example, probabilistic range queries are the focus of [38], and probabilistic nearest neighbor queries are studied in [7, 26]. In [45], the authors present a model to index uncertain objects and solve probabilistic queries.

In particular, as we mentioned in Section 2.2, the processing of probabilistic inside query has been studied previously, 
LB-5 Filtering Rate

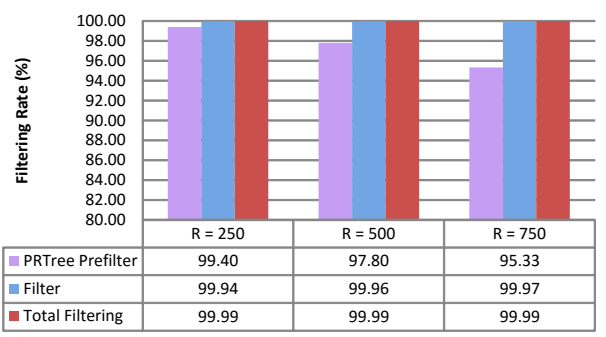

LB-100 Filtering Rate

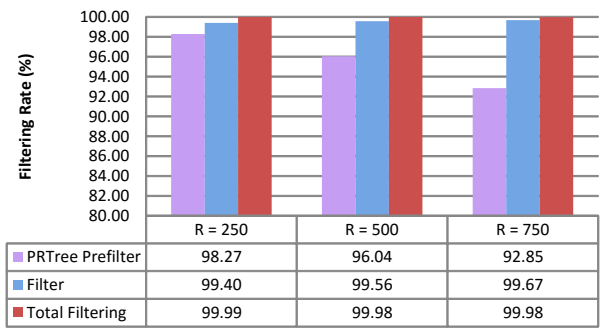

LB-250 Filtering Rate

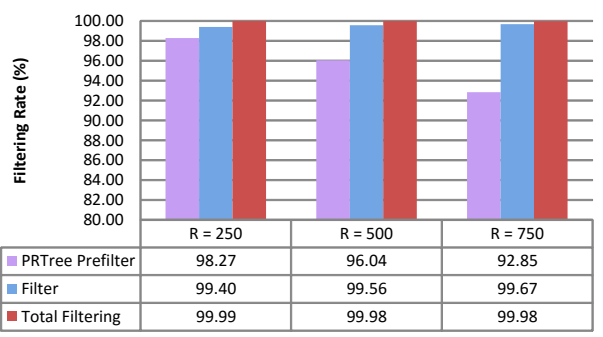

Figure 15: Filtering rate for the different uncertainty values (LB-5,LB-100,LB-250) in the LB dataset experiments.

LB - No indexed - PC

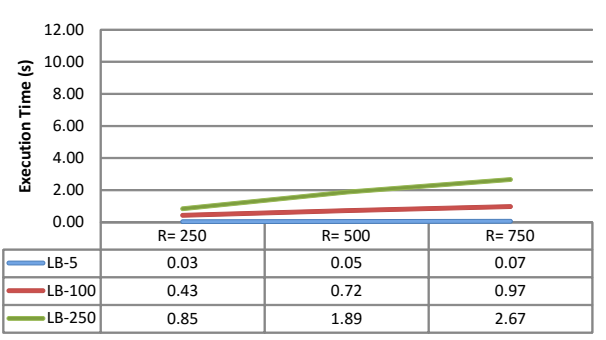

LB - Indexed - PC

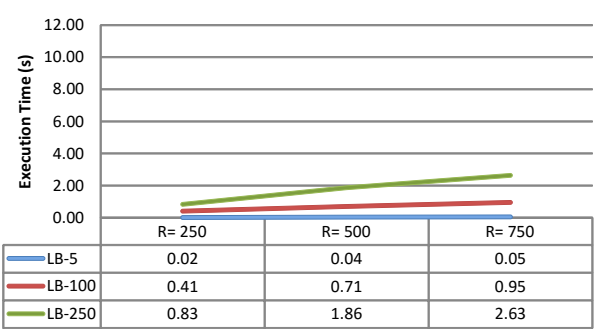

LB - No indexed - Android - SG

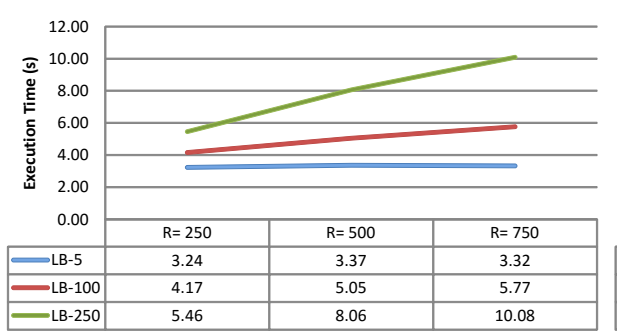

LB - Indexed - Android - SG

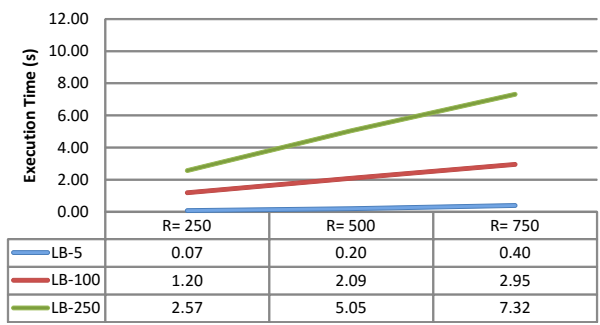

LB - No indexed - Android - BQ M5

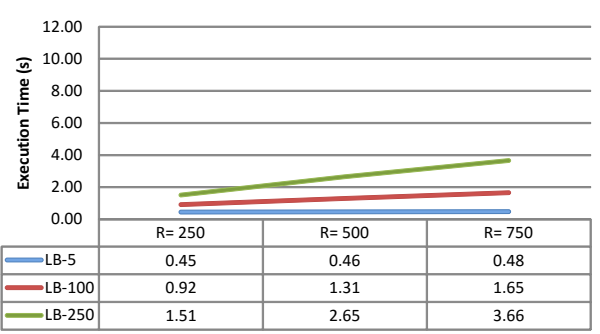

LB - Indexed - Android - BQ M5

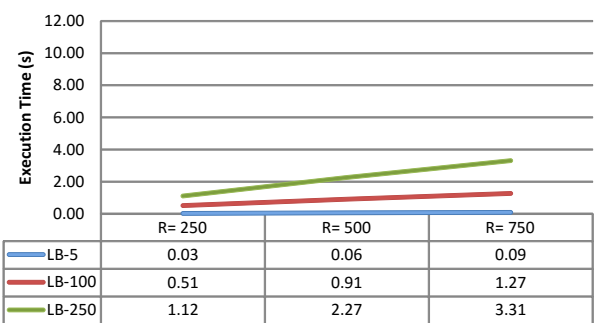

Figure 16: Performance of our approach for the different uncertainty values (LB-5,LB-100,LB-250) on a PC and two Android devices.

mainly, in $[1,38,44,45]$. In all these works, the bottleneck in the performance is the calculus of numerical integrals. So, the fewer integrals have to be calculated, the better is the performance. In [38], the authors propose an index, called U-tree, that avoids doing some of these integrals. The U-tree is an $\mathrm{R}^{*}$-tree augmented with some information about the $p d f$ of the uncertain objects. This information is based on PCRs (probabilistically constrained rectangles), an extension of the concept of minimum bounding rectangles for uncertain areas. The filter capabilities using the U-tree behave quite well when the range query is a rectangle, but it has worse results when the range query is a circle, as we have in our experiments. Trying to overcome this problem, the authors in [44] construct an index, a UI-tree, which is also a modified R-tree. In this case, the UItree holds some disjoint partitions of the uncertain area of the objects together with the cumulative $p d f$ in each of these partitions. A different method is proposed in [1], where the authors define an index, called UP-tree, based on the selection of some pivot points that are used to prune (not validate) objects by the reverse triangle inequality. Finally, in [45], the authors define a U-Quadtree, a Quadtree with additional information about the $p d f$ of objects. The main difference with the other methods is that the summary information about the $p d f$ saved on the index depends on where the $p d f$ has more information.

The previous approaches do not constrain the used $p d f s$; however, they rely on the assumption that pre-indexing the objects is always feasible, which might not be the case in a mobile and volatile environment. Moreover, as shown in the experimental section, our approach would be complementary to their works in order to lower the number of numeric integrals to be solved.

There are several works $[6,9,13,24,28,31,46]$ which consider different types of probabilistic queries (e.g., different variants of nearest neighbour queries and range queries within temporal ranges and trajectories). However, none of them, but for [6], consider that the uncertainty can also affect the queried position (e.g., the query could be expressed in terms also of a moving object, and thus, be subject of uncertainty). The work in [6] focuses on calculating the similarity domination rather than the type query that we are dealing with in our work.

Thus, as opposed to existing related work, in this paper we have presented a model of uncertainty based on the concept of location granule, analyzed the problem of probabilistic inside queries using it, and proposed a different (and complementary 
to other related works) approach to speed up the calculations needed to solve probabilistic inside queries, where the uniform distribution is considered and which filters a large percentage of the objects of the search space by just performing a few operations.

\section{Conclusions and Future Work}

Location-dependent queries are the main building block of location-based services, and therefore we have to provide efficient methods to solve them. However, as we have seen, they usually do not take into account the imprecision of the locations (inherent to the mechanism or introduced to force it, for example, for privacy issues). When you consider imprecise locations, the uncertainty in object locations also introduce a heavyweight problem when solving the already troublesome locationdependent constraints.

In this paper, we have presented an extension of our location model $[5,21]$ to deal with location imprecision using uncertainty location granules, thus combining the higher semantics achieved thanks to the use of location granules with probabilistic approaches in a seamless way. In particular:

- We have formalized the concept of uncertainty granule to model uncertain locations and their relationship with traditional location granules (granules without uncertainty). This has allowed to extend our location granule model to deal with imprecise locations in a seamless way.

- We have analyzed a type of probabilistic inside query, and provided a method to solve them efficiently when the underlying probability density function is the uniform distribution.

- We have performed an extensive experimental evaluation with both synthetic and real datasets where the efficiency of the described method for the uniform distribution can be appreciated. The results obtained showed the interest of our proposal, even in non-indexed and mobile scenarios (where the calculations could be performed in mobile devices).

We are currently studying how to extend our method for normal $p d f s$ and histograms (i.e., how to calculate rapidly an approximation of the integral using annuli), and how our approach could be applied and integrated with other approaches based on indexing. We also plan to study how the use of different location granularities would affect the semantics of probabilistic inside constraints and their efficient calculation. Last but not least, we will study other popular location-dependent constraints (such as nearest-neighbor queries, closest-pairs [14, 15] and similarity joins [14], as well as reverse kNN queries [37]) .

\section{Acknowledgments}

This work was supported by the CICYT project TIN201346238-C4-4-R and DGA-FSE.
[1] F. Angiulli, F. Fassetti, Indexing uncertain data in general metric spaces, IEEE Transactions on Knowledge Data Engineering 24 (9) (2012) 16401657.

[2] L. Arge, M. D. Berg, H. Haverkort, K. Yi, The priority R-tree: A practically efficient and worst-case optimal R-tree, ACM Transactions on Algorithms 4 (1) (2008) 9:1-9:30.

[3] P. Bellavista, A. Corradi, C. Giannelli, Efficiently managing location information with privacy requirements in Wi-Fi networks: a middleware approach, in: 2nd International Symposium on Wireless Communication Systems (ISWCS'05), Siena, Italy, 2005, pp. 91-95.

[4] A. Belussi, C. Combi, G. Pozzani, Formal and conceptual modeling of spatio-temporal granularities, in: International Database Engineering \& Applications Symposium (IDEAS'09), Cetraro, Calabria, Italy, 2009, pp. 275-283.

[5] J. Bernad, C. Bobed, E. Mena, S. Ilarri, A formalization for semantic location granules, International Journal of Geographical Information Science 27 (6) (2013) 1090-1108.

[6] T. Bernecker, T. Emrich, H.-P. Kriegel, N. Mamoulis, M. Renz, A. Züfle, A novel probabilistic pruning approach to speed up similarity queries in uncertain databases, in: IEEE 27th International Conference on Data Engineering (ICDE'11), Hannover, Germany, 2011, pp. 339-350.

[7] G. Beskales, M. A. Soliman, I. F. Ilyas, Efficient search for the top-k probable nearest neighbors in uncertain databases, Proceedings of the VLDB Endowment 1 (1) (2008) 326-339.

[8] Y. Cai, K. A. Hua, G. Cao, T. Xu, Real-time processing of rangemonitoring queries in heterogeneous mobile databases, IEEE Transactions on Mobile Computing 5 (7) (2006) 931-942.

[9] R. Cheng, J. Chen, M. Mokbel, C. Y. Chow, Probabilistic verifiers: Evaluating constrained nearest-neighbor queries over uncertain data, in: IEEE 24th International Conference on Data Engineering (ICDE'08), Cancun, Mexico, 2008, pp. 973-982.

[10] R. Cheng, D. V. Kalashnikov, S. Prabhakar, Evaluating probabilistic queries over imprecise data, in: ACM SIGMOD International Conference on Management of Data (SIGMOD'03), San Diego, California, USA, 2003, pp. 551-562.

[11] R. Cheng, D. V. Kalashnikov, S. Prabhakar, Querying imprecise data in moving object environments, IEEE Transactions on Knowledge and Data Engineering 16 (9) (2004) 1112-1127.

[12] C.-Y. Chow, M. F. Mokbel, H. V. Leong, On efficient and scalable support of continuous queries in mobile peer-to-peer environments, IEEE Transactions on Mobile Computing 10 (10) (2011) 1473-1487.

[13] B. S. Chung, W.-C. Lee, A. L. Chen, Processing probabilistic spatiotemporal range queries over moving objects with uncertainty, in: 12th International Conference on Extending Database Technology (EDBT'09), Saint-Petersburg, Russia, 2009, pp. 60-71.

[14] A. Corral, Y. Manolopoulos, Y. Theodoridis, M. Vassilakopoulos, Closest pair queries in spatial databases, in: ACM SIGMOD International Conference on Management of Data (SIGMOD'00), Dallas, Texas, USA, 2000, pp. 189-200.

[15] A. Corral, M. Vassilakopoulos, On approximate algorithms for distancebased queries using R-trees, The Computer Journal 48 (2) (2005) 220238.

[16] H. Ding, G. Trajcevski, P. Scheuermann, Efficient maintenance of continuous queries for trajectories, Geoinformatica 12 (3) (2008) 255-288.

[17] B. Gedik, L. Liu, MobiEyes: A distributed location monitoring service using moving location queries, IEEE Transactions on Mobile Computing 5 (10) (2006) 1384-1402.

[18] J. Hightower, From position to place, in: 2003 Workshop on LocationAware Computing, Seattle, Washington, USA, 2003, pp. 10-12.

[19] C. Hoareau, I. Satoh, A model checking-based approach for location query processing in pervasive computing environments, in: On the Move to Meaningful Internet Systems 2007: OTM 2007 Workshops (PerSys'07), Vilamoura, Algarve, Portugal, vol. 4806 of Lecture Notes in Computer Science (LNCS), 2007, pp. 866-875.

[20] C. Hoareau, I. Satoh, From model checking to data management in pervasive computing: a location-based query-processing framework, in: International Conference on Pervasive Services (ICPS'09), London, United Kingdom, 2009, pp. 41-48.

[21] S. Ilarri, C. Bobed, E. Mena, An approach to process continuous locationdependent queries on moving objects with support for location granules, Journal of Systems and Software 84 (8) (2011) 1327-1350. 
[22] S. Ilarri, E. Mena, A. Illarramendi, Location-dependent queries in mobile contexts: Distributed processing using mobile agents, IEEE Transactions on Mobile Computing 5 (8) (2006) 1029-1043.

[23] S. Ilarri, E. Mena, A. Illarramendi, Location-dependent query processing: Where we are and where we are heading, ACM Computing Surveys 42 (3) (2010) 12:1-12:73

[24] Y. Jin, R. Cheng, B. Kao, K.-Y. Lam, Y. Zhang, A filter-based protocol for continuous queries over imprecise location data, in: 21st ACM International Conference on Information and Knowledge Management (CIKM'12), Maui, Hawaii, USA, 2012, pp. 365-374.

[25] H.-P. Kriegel, P. Kunath, M. Pfeifle, M. Renz, Probabilistic similarity join on uncertain data, in: 11th International Conference on Database Systems for Advanced Applications (DASFAA'06), Singapore, 2006, pp. 295-309.

[26] H.-P. Kriegel, P. Kunath, M. Renz, Probabilistic nearest-neighbor query on uncertain objects, in: 12th International Conference on Database Systems for Advanced Applications (DASFAA'07), Bangkok, Thailand, 2007, pp. 337-348.

[27] J. Krumm, A survey of computational location privacy, Personal and Ubiquitous Computing 13 (6) (2009) 391-399.

[28] E.-Y. Lee, H.-J. Cho, T.-S. Chung, K.-Y. Ryu, Moving range k nearest neighbor queries with quality guarantee over uncertain moving objects, Information Sciences 325 (2015) 324 - 341

[29] C.-Y. Lin, Y.-C. Tseng, Y.-C. Liu, Imprecision-tolerant location management for object-tracking wireless sensor network, The Computer Journal 53 (3) (2010) 351-364.

[30] M. F. Mokbel, X. Xiong, M. A. Hammad, W. G. Aref, Continuous query processing of spatio-temporal data streams in PLACE, Geoinformatica 9 (4) (2005) 343-365.

[31] J. Niedermayer, A. Züfle, T. Emrich, M. Renz, N. Mamoulis, L. Chen, H.-P. Kriegel, Probabilistic nearest neighbor queries on uncertain moving object trajectories, Proceedings of the VLDB Endowment 7 (3) (2013) 205-216.

[32] E. Pitoura, I. Fudos, Distributed location databases for tracking highly mobile objects, The Computer Journal 44 (2) (2001) 75-91.

[33] S. Prabhakar, Y. Xia, D. V. Kalashnikov, W. G. Aref, S. E. Hambrusch, Query indexing and velocity constrained indexing: Scalable techniques for continuous queries on moving objects, IEEE Transactions on Com- puters 51 (10) (2002) 1124-1140.

[34] J. H. Schiller, A. Voisard, Location-Based Services, Morgan Kaufmann, San Francisco, California, USA, 2004

[35] A. P. Sistla, O. Wolfson, S. Chamberlain, S. Dao, Modeling and querying moving objects, in: IEEE 13th International Conference on Data Engineering (ICDE'97), Birmingham, United Kingdom, 1997, pp. 422-432.

[36] Y. Sun, T. F. La Porta, P. Kermani, A flexible privacy-enhanced locationbased services system framework and practice, IEEE Transactions on Mobile Computing 8 (3) (2009) 304-321.

[37] D. Taniar, M. Safar, Q. T. Tran, W. Rahayu, J. H. Park, Spatial network RNN queries in GIS, The Computer Journal 54 (4) (2011) 617-627.

[38] Y. Tao, X. Xiao, R. Cheng, Range search on multidimensional uncertain data, ACM Transactions on Database Systems 32 (3) (2007) 15:1-15:54.

[39] Y. Theodoridis, Ten benchmark database queries for location-based services, The Computer Journal 46 (6) (2003) 713-725.

[40] E. Trevisani, A. Vitaletti, Cell-ID location technique, limits and benefits: An experimental study, in: 6th IEEE Workshop on Mobile Computing Systems and Applications (WMCSA'04), Washington, DC, USA, 2004, pp. 51-60.

[41] M. van Kreveld, Geo-information and computational geometry, chap. 1. Computational geometry: Its objectives and relation to GIS, Nederlandse Commissie voor Geodesie (NCG), 2006, pp. 1-8.

[42] T. Yoshimura, H. Hasegawa, Comparing the precision and accuracy of GPS positioning in forested areas, Journal of Forest Research 8 (3) (2003) $147-152$.

[43] V. Zeimpekis, G. M. Giaglis, G. Lekakos, A taxonomy of indoor and outdoor positioning techniques for mobile location services, SIGecom Exchanges 3 (4) (2002) 19-27.

[44] Y. Zhang, X. Lin, W. Zhang, J. Wang, Q. Lin, Effectively indexing the uncertain space, IEEE Transactions on Knowledge and Data Engineering 22 (9) (2010) 1247-1261.

[45] Y. Zhang, W. Zhang, Q. Lin, X. Lin, H. T. Shen, Effectively indexing the multidimensional uncertain objects, IEEE Transactions on Knowledge and Data Engineering 26 (3) (2014) 608-622.

[46] K. Zheng, G. Trajcevski, X. Zhou, P. Scheuermann, Probabilistic range queries for uncertain trajectories on road networks, in: 14th International Conference on Extending Database Technology (EDBT'11), Uppsala, Sweden, 2011, pp. 283-294. 